\title{
Radio Wave Absorption of Several Gases in the 100 to 117 kMc/s Frequency Range
}

\author{
C. O. Britt, C. W. Tolbert, and A. W. Straiton
}

(May 16, 1960; revised June 23, 1960)

\begin{abstract}
This paper describes a technique for the measurement of the absorption characteristics of millimeter radio waves over a wide pressure range and presents the results of measurements made with this technique for four gases in the frequency band from 100,000 to 117,000 megacycles per second. The four gases investigated in near pure state were carbon monoxide, nitrous oxide, sulfur dioxide, and nitrogen dioxide. The results of these tests are as follows:
\end{abstract}

\begin{tabular}{|c|c|c|c|c|}
\hline Gas & $\begin{array}{l}\text { Predicted or } \\
\text { previously } \\
\text { measured } \\
\text { frequency }\end{array}$ & $\begin{array}{l}\text { New measure- } \\
\text { ment of frequency }\end{array}$ & $\begin{array}{c}\text { Measured } \\
\text { absorption } \\
\text { coefficient }(\alpha)\end{array}$ & $\begin{array}{l}\text { Meas- } \\
\text { ured line } \\
\text { width }\end{array}$ \\
\hline \multirow{4}{*}{$\begin{array}{l}\text { Carbon monoxide... } \\
\text { Nitrous oxide. } \\
\text { Sulfur dioxide. }\end{array}$} & $M c / s$ & $M c / s$ & $\frac{c m^{-1}}{2}$ & $\begin{array}{l}\mathrm{Mc} / \mathrm{s} \text { per } \\
\mathrm{mm} \mathrm{Hg}\end{array}$ \\
\hline & $115,270.56$ & \multirow{3}{*}{$\begin{array}{l}104,042 \text { and } \\
104,046 . \\
105,189\end{array}$} & $8.75 \times 10^{-5}$ & \multirow{3}{*}{$\begin{array}{r}4.25 \\
4.25 \\
13.75 \\
17.0\end{array}$} \\
\hline & $100,491.76$ & & 1. $25 \times 10^{-4}$ & \\
\hline & $105,333.94$ & & $9 \times 10^{-6}$ & \\
\hline Nitrogen dioxide..... & $105,158.4$ & No & e detected & \\
\hline
\end{tabular}

\section{Introduction}

The Electrical Engineering Research Laboratory of the University of Texas has made a number of measurements throughout the low-centimeter and millimeter wavelength regions of the absorption of radio waves due to atmospheric gases [1]. ${ }^{2}$ By repeating these tests, the attenuation was plotted as a function of water vapor content. In almost every instance, it was possible to interpret the losses as being due to absorption by water vapor and oxygen.

In one set of measurements, however, variable losses were noted which were not associated with changes in water vapor concentration. These losses occurred in the frequency range between 100,000 and $118,000 \mathrm{Mc} / \mathrm{s}$ and were attributed to some of the rarer gases in the atmosphere [2].

It is the purpose of this present paper to investigate the absorption properties of several gases with measured or predicted absorption lines in the frequency region in question. In order to isolate the effect of each line, the gases were studied in the nearly pure state. The absorption characteristics obtained by measuring the gases individually cannot immediately be used to predict their absorption when diluted with air. Further studies are in progress which, we bope, will assist in making this transition from individual gas loss to loss due to mixtures.

1 Contribution from Electrical Engineering Research Laboratory, The University of Texas, Austin, Tex. Prepared under Office of Naval Research Contract Nonr 375(01), NR 371032.

2 Figures in brackets indicate the literature references at the end of this paper.

\section{Instrumentation and Measuring Technique}

The millimeter wave absorption was measured by the use of a circular tube $500 \mathrm{ft}$ in length and $6 \frac{1}{8}$ in. in diameter. The cell may be evacuated and then filled with the gas under test. For the measurement of the larger absorption coefficients, a diaphragm was inserted between sections of the pipe and only part of the length was filled with the test gas. The remainder of the tube was filled with nonabsorbing gas.

A Teflon diaphragm at either end permitted the injection and extraction of the electromagnetic energy. Horn antennas spaced back of the ends of the pipe provided proper illumination across the diaphragms. The electric field across the end of the cell was in a vertical direction with the intensity varying horizontally from maximum at the center line to zero at the edges. This field approximates that of the $\mathrm{TE}_{11}$ mode. Distortion from higher-order modes was very small since the attenuation in the guide for lossless gases was only $1 / 4 \mathrm{db}$.

The signal source consisted of a Bell Telephone Laboratories M-1805 klystron operating over a 50,000 - to $58,500-\mathrm{Mc} / \mathrm{s}$ range, a modified L-5506 "in-guide" crystal harmonic generator to provide the second harmonic for the tests, and the associated power supplies and necessary waveguide components. The maximum signal available was of the order of $10^{-7} \mathrm{~W}$.

The receiver also utilizes an M-1805 klystron, an "in-guide" wafer crystal for harmonic generation and mixing, and the necessary amplifiers and power supplies. The receiver was frequency modulated to reduce the d-c amplifier requirements and to compensate for klystron frequency instability.

The usual measurement procedure was to evacuate the cell and determine the attenuation at the frequencies of interest as the test gas pressure was increased. A continuous frequency scanning technique was used at low pressures in the vicinity of the maximum absorption. 


\section{Carbon Monoxide}

As is the case of each of the various gases studied, the frequencies of the absorption lines of carbon monoxide had been predicted from a study of the infrared molecular energy levels. The various resonant frequencies have been tabulated by Ghosh and Edwards [3]. Gordy and his associates have measured with great accuracy the frequency of the carbon monoxide line in the spectrum of interest in this paper. A comparison of these values is as follows:

Predicted:

$115,270.2 \mathrm{Mc} / \mathrm{s}$

Measured (Gordy): $\quad 115,270.56 \mathrm{Mc} / \mathrm{s}$.

Equipment was not available at this laboratory for absolute frequency measurements to this precision. The above absorption line at the upper end of the band and one for nitrous oxide at the lower end of the band, which was also measured with accuracy by Gordy, were used as calibration points on the wavemeter. By assuming linear variation between these points, a measure was obtained of the intermediate frequencies.

The absorption versus frequency measurements in the vicinity of the carbon monoxide line are shown in figure 1. ${ }^{3}$ Pressures ranging from 4.5 to $27 \mathrm{~mm}$ of mercury were used in obtaining these data. The lines drawn on figure 1 were calculated by the

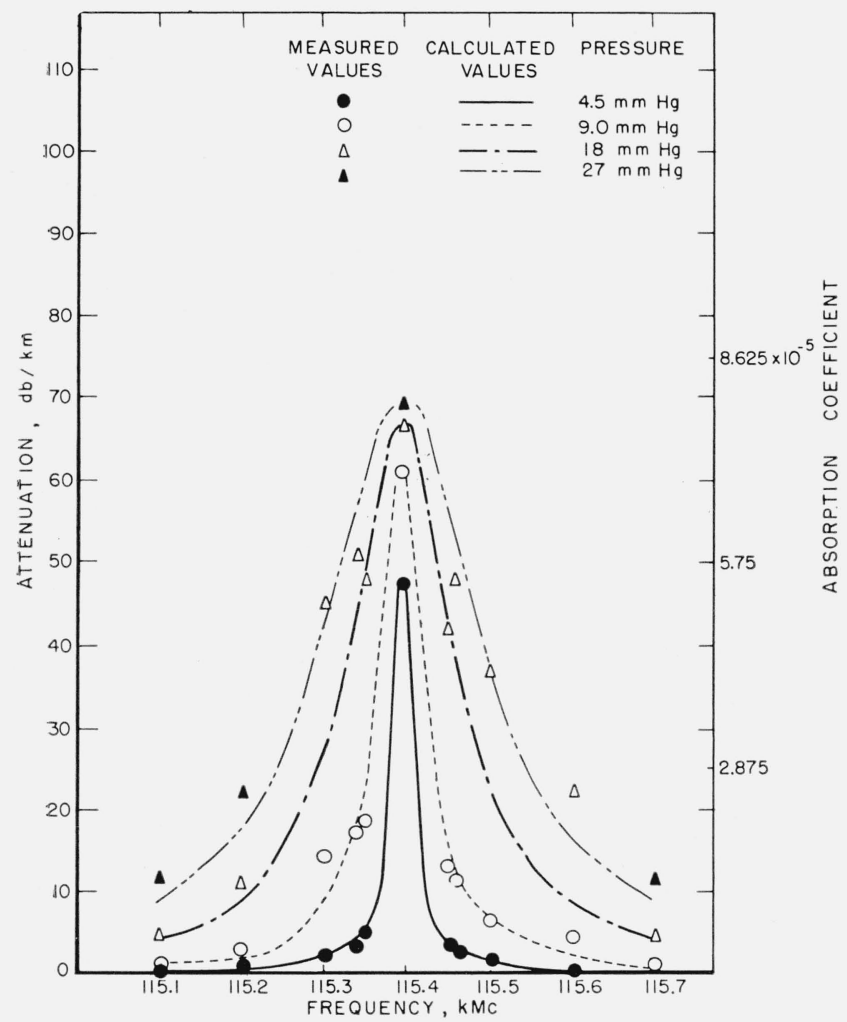

Figure 1. Carbon monoxide absorption spectra.

Absorption coefficient as used in the figures is in nepers per centimeter. use of the Van Vleck-Weisskopf aquation [5] with the maximum attenuation in the equation being adjusted to fit the maximum measured value at the pressure in question. A line breadth proportional to pressure with a value of $4.25 \mathrm{Mc} / \mathrm{s}$ per mm $\mathrm{Hg}$ was obtained from the half power points at very low pressure where continuous sweep of the frequency was used. This line breadth constant provided a fairly good fit of the measured data when the maximum absorption was adjusted to fit the measured maximum at each pressure. This variation of maximum absorption with pressure is not implied in the Van Vleck-Weisskopf equation, but it may possibly be explained by saturation effects [6]. This decrease of absorption at the lower pressures has been attributed to the power saturation of the molecules and the possibility that lines could not be completely resolved due to a small amount of frequency modulation present in the measuring signal. Power variations over a range of several decibels did not, however, seem to alter materially the shape of the maximum absorption-pressure curve.

To further investigate the pressure dependence of the absorption, this peak attenuation was measured over a pressure range from a few millimeters to nearly $1 \mathrm{~atm}$. These data are shown in figure 2. The absorption is seen to increase rapidly at very low pressures and slowly at higher pressures. The higher pressure increase is thought to be due to the "skirts" of higher frequency lines. The absorption coefficient, $\alpha$, is chosen as the value at which the curvature changes abruptly since the gradual increase with pressure above the knee of the curve is attributed to contributions from other lines. The saturation effect is assumed to be confined to the very low pressures.

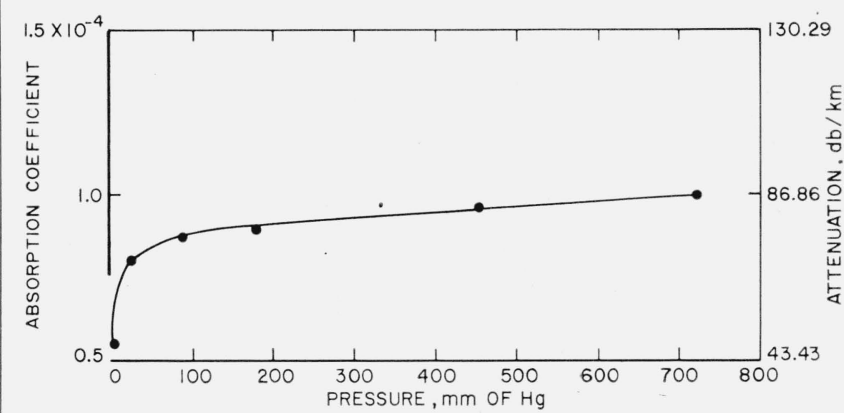

Figure 2. Absorption versus pressure for carbon monoxide. Measured values.

\section{Nitrous Oxide}

Gordy and his associates [4] had measured with precision the frequency of the nitrous oxide line predicted for the spectrum covered by this report. A comparison of the measured and predicted values is as follows:
Predicted:
Measured (Gordy): 
As indicated earlier, the frequency of this line was used as a reference in the calibration of the wavemeter for the frequency range in question.

The nitrous oxide absorption spectra for a number of pressures are shown in figure 3. The curves in the figure were drawn using the Van Vleck-Weisskopf equation with a line breadth proportional to pressure for a proportionality constant of $4.25 \mathrm{Mc} / \mathrm{s}$ per $\mathrm{mm}$ $\mathrm{Hg}$. This line breadth constant was also determined from the half-power width at very low pressures. As in the case of carbon monoxide, it was necessary to adjust the peak absorption for the particular pressure used.

The variation of the peak absorption as a function of pressure for nitrous oxide is shown in figure 4 .

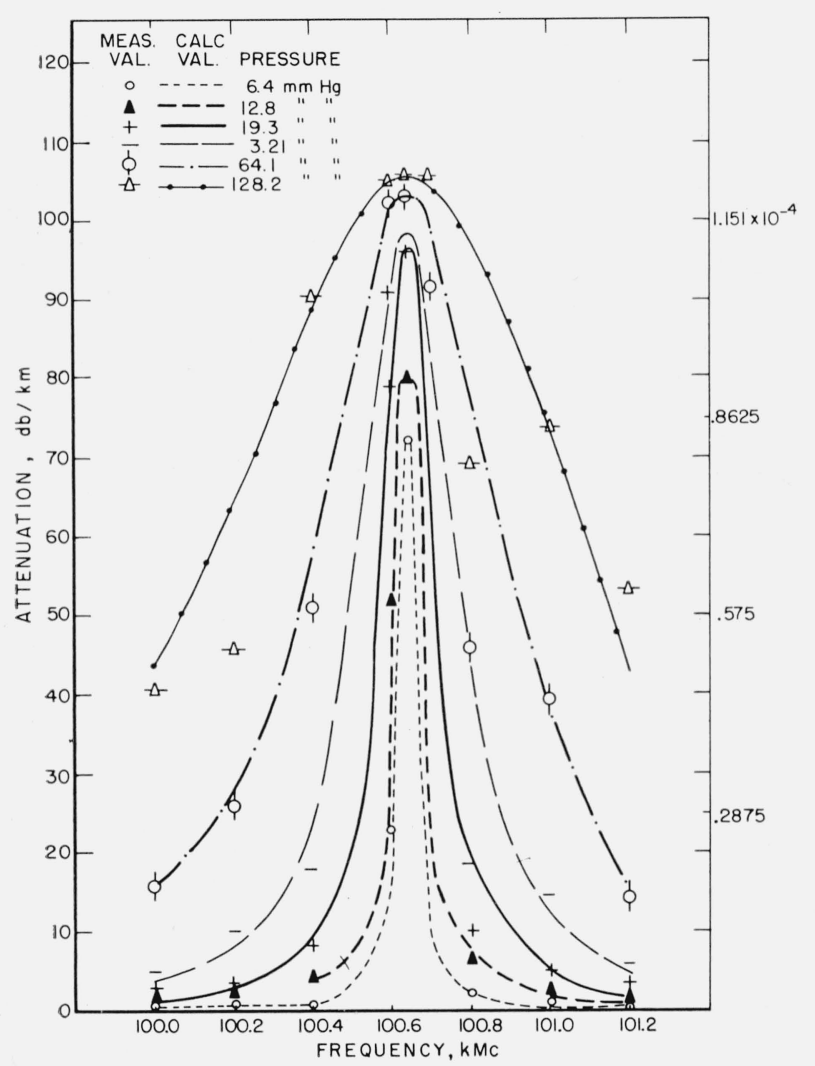

Figure 3. Nitrous oxide absorption spectra.

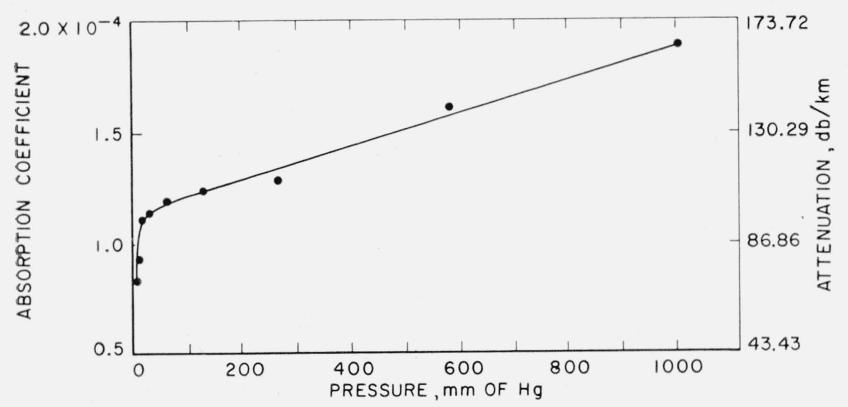

Figure 4. Absorption versus pressure for nitrous oxide.
The low-pressure change may be $\mathrm{f}$-plained in part by saturation effects and the hig! - pressure change by the effect of the skirts of other 'ines. The absorption coefficient applicable to the l.ne is chosen as the value at the point of most rapir, change in slope as in the case of carbon monoxide.

\section{Sulfur Dioxide}

Ghosh and Edwards [3] list four predicted sulfur dioxide lines in the vicinity of the spectrum covered by this report as follows:

$$
\begin{aligned}
& 100,911.75 \\
& 104,028.28 \\
& 105,333.94 \\
& 116,790.07
\end{aligned}
$$

plus 40 additional lines between 44,000 and 200,000 $\mathrm{Mc} / \mathrm{s}$.

The sulfur dioxide spectra were examined in detail over the range from 102,000 to $106,000 \mathrm{Mc} / \mathrm{s}$ and the results are shown in figure 5. The effects of the first three lines listed above are readily seen in this figure. In addition, the complex spectra of sulfur dioxide was evident in the flattening of the absorption spectra over the region investigated.

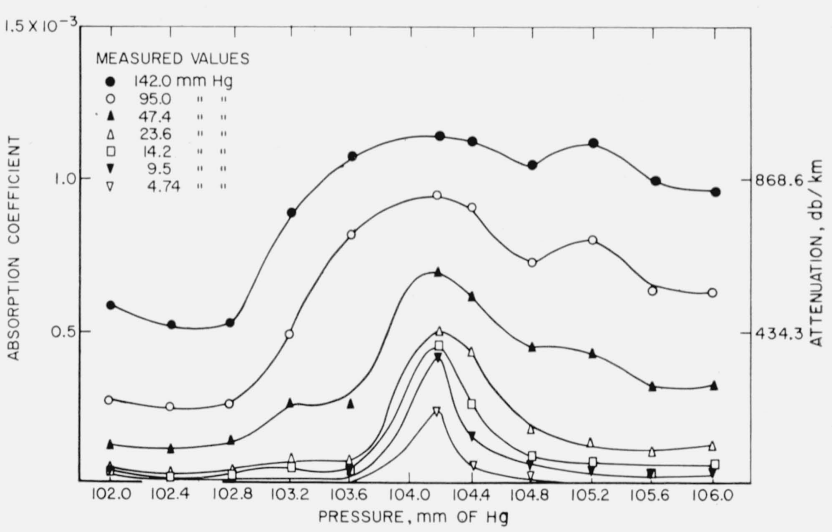

Figure 5. Absorption coefficient of sulfur dioxide versus frequency at different pressures.

The line in the vicinity of $104,000 \mathrm{Mc} / \mathrm{s}$ was examined at low pressures by frequency scanning and was found to consist of two lines with a separation of $4.5 \mathrm{Mc} / \mathrm{s}$ centered approximately at 104,042 and $104,046 \mathrm{Mc} / \mathrm{s}$. Each line had a width of 13.7 $\mathrm{Mc} / \mathrm{s}$ per $\mathrm{mm} \mathrm{Hg}$ and the absorption of lower frequency component was approximately 1.4 times more intense than the higher frequency one.

The maximum absorption of the combined line as it blended together at increased pressures was measured and is shown in figure 6 . The lower line in this figure is the absorption which would be associated with the double line in the vicinity of 104,000 $\mathrm{Mc} / \mathrm{s}$. The frequency for which the calculations were made falls between the two lines and therefore goes essentially to zero at very low pressures. The difference in the measured and calculated values is 


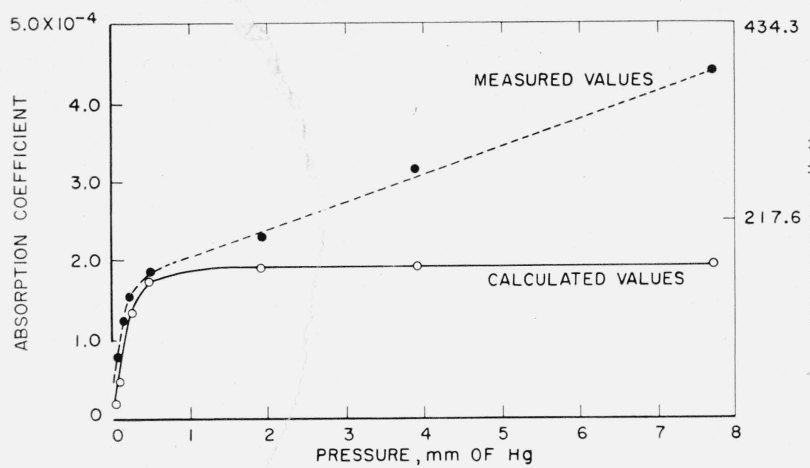

FiguRE 6. Maximum absorption coefficient for two observed lines of sulfur dioxide versus pressure.

apparently due to the attenuation caused by the skirts of the many other lines. The absorption of the 105,189 line was so low that it could not be resolved at the lower pressures. Its effect is evident, however, at higher pressures (fig. 5).

\section{Nitrogen Dioxide}

An absorption line had been predicted at a frequency of $105,158.4 \mathrm{Mc} / \mathrm{s}$ for nitrogen dioxide. A continuous search was made from 104,200 to 105,600 $\mathrm{Mc} / \mathrm{s}$ at very low pressures and spot measurements were made at $104,720,104,920$, and $105,120 \mathrm{Mc} / \mathrm{s}$. In none of the tests was there any indication of appreciable attenuation. It was felt that if a line of nitrogen dioxide exists with significant strength of absorption, its effect would have been noted.

\section{Conclusions}

The measurements described in this paper have confirmed the presence of absorption lines for carbon monoxide, nitrous oxide, and sulfur dioxide in the frequency range from 100,000 to $117,000 \mathrm{Mc} / \mathrm{s}$. A predicted line for nitrogen dioxide could not be detected. The data can be represented by the Van
Vleck-Weisskopf [5] line shape function using a line breadth proportional to pressure and a maximum loss at a given pressure adjusted to fit the measured maximum. One of the predicted lines for sulfur dioxide was found to be a doublet. The numerical results are summarized in the following table:

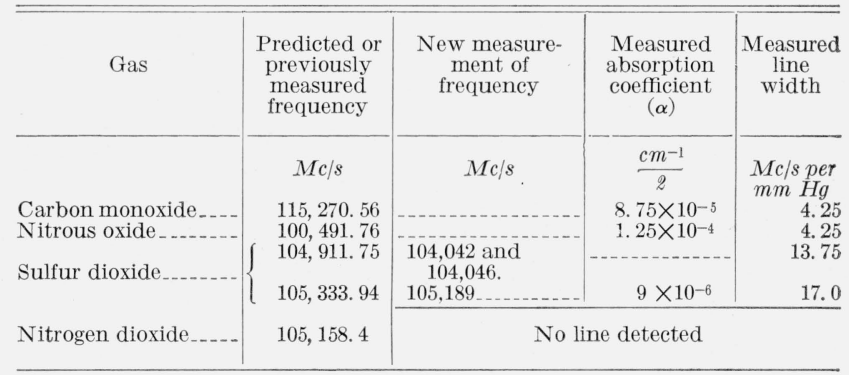

\section{References}

[1] A. W. Straiton and C. W. Tolbert, Anomalies in the absorption of radio waves by atmospheric gases, Proc. IRE 48, 898 (1960).

[2] C. W. Tolbert and A. W. Straiton, Radio propagation measurements in the 100 to 118 Kmes spectrum, IRE Wescon Conv. Record, Pt. 1, pp. 56-44 (Aug. 1959).

[3] S. N. Ghosh and H. D. Edwards, Rotational frequencies and absorption coefficients of atmospheric gases, Air Force Survey in Geophys., No. 82, Air Force Cambridge Research Center (March 1956).

[4] C. M. Johnson, R. F. Trambarulo, and W. Gordy, Microwave spectroscopy in the region from two to three millimeters, Pt. II, Phys. Rev. 84, 1178 (1951).

[5] J. H. Van Vleck and V. Weisskopf, On the shape of collision-broadening lines, Rev. Mod. Phys. 1\%, 17 (1949).

[6] W. Gordon, W. V. Smith, and R. F. Trambarulo, Microwave spectroscopy, p. 197 (John Wiley \& Sons, Inc., New York, N.Y., 1953). 